\title{
Simulation of kinematics of SS 433 radio jets that interact with the ambient medium
}

\author{
А. A. Panferov (Панфёров, Александр Анатольевич)
}

\begin{abstract}
Institute of Mathematics, Physics and Information Technologies (IMFIT), Togliatti State University, Belorusskaya 14, 445667, Russia e-mail: panfS@yandex.ru
\end{abstract}

Received 7 August 2013 / Accepted 8 January 2014

\begin{abstract}
Context. The mildly relativistic jets of SS 433 are believed to inflate the surrounding supernova remnant W 50, possibly depositing more than $99 \%$ of their kinetic energy in the remnant expansion. Where and how this transformation of the energy occurs is as yet unknown. We can learn from this that the jets decelerate and that this deceleration is non-dissipative.

Aims. We uncover the deviation of the arcsecond-scale precessing radio jets of SS 433 from the ballistic locus described by the kinematic model as a signature of the dynamics issuing from the interaction of the jets with the ambient medium.

Methods. To do this, we simulated the kinematics of these jets, taking into account the ram pressure on the jets, which we estimated from the profile of brightness of synchrotron radiation along the radio jets, assuming pressure balance in the jets.

Results. We found that to fit an observable locus in all scales the radio jets need to be decelerated and twisted in addition to the precession torsion, mostly within the first one-fifth of the precession period, and subsequently they extend in a way that imitates ballistic jets. This jet kinematics implies a smaller distance to SS 433 than the currently accepted $5.5 \mathrm{kpc}$. The physical parameters of the jet model, which links jets dynamics with radiation, are physically reliable and characteristic for the SS 433 jets. The model proposes that beyond the radio-brightening zone, the jet clouds expand because they are in pressure balance with the intercloud medium, and heat up with distance according to the law $T=2 \times 10^{4}\left(r / 10^{15} \mathrm{~cm}\right)^{1.5} \mathrm{~K}$.

Conclusions. This model naturally explains and agrees with, the observed properties of the radio jets: a) the shock-pressed morphology; b) the brightness profile; c) the $\sim 10 \%$ deflections of the jet kinematics from the standard kinematic model - a magnitude of the jet speed decrement in our simulation; d) the precession-phase deviations from the standard kinematic model predictions; e) the dichotomy of the distances to the object, $4.8 \mathrm{kpc}$ vs. $5.5 \mathrm{kpc}$, which are determined on the basis of the jet kinematics on scales of sub-arcsecond and several arcseconds, respectively; and f) the reheating on arcsecond scales.
\end{abstract}

Key words. binaries: close - stars: individual: SS 433 - stars: jets - ISM: jets and outflows

\section{Introduction}

The unique binary star SS 433 is one of the most energetic stars of our Galaxy (see review of Fabrika, 2004). Its radiative luminosity, $\gtrsim 10^{40} \mathrm{erg} / \mathrm{s}$, is determined by a supercritical accretion disk around a compact star, probably a black hole. The anisotropy of the disk radiation flux, with the maximum along the disk axis, suggests that SS 433 represents a whole class of objects of still unknown nature - ultraluminous X-ray sources in other galaxies - that are presumably observed close to the disk axis (e.g. Begelman et al., 2006). In addition, the two symmetric mildly relativistic jets of SS 433 are brilliant representatives of astrophysical jets. With these properties SS 433 is a stellar analogue of active galactic nuclei (a quasar vice versa).

In the radio the SS 433 jets are viewed at distances from the jets source from sub-milliarcsecond to some arcseconds ${ }^{1}$ as conical corkscrews of a semi-opening $\theta_{\mathrm{pr}} \approx 20^{\circ}-$ a signature of jet precession. After this, the jets are unseen and appear only on scales of sub-degree in X-ray emission along the precession axis and in the weak shock waves in the lobes at the intersection of the axis with the shell of supernova remnant W 50 encircling SS 433.

Kinematics of the radio jets as well as Doppler shifts of spectral lines of the X-ray and optical jets, which radiate at distances

$1^{\prime \prime}=0.72 \times 10^{17} \mathrm{~cm}$ at a distance of $4.8 \mathrm{kpc}$. for the source of the jets $r \sim 10^{11} \mathrm{~cm}$ and $\sim 10^{15} \mathrm{~cm}$, respectively, fulfils the same (standard) kinematic model, according to which a jet axis precesses and nutates, and each element of the jet moves free (so-called ballistic movement; Abell \& Margon, 1979; Hjellming \& Johnston, 1981; Newsom \& Collins, 1981). Though the radio jets at their origin are intimately connected with the optical jets, which are believed to be ballistic (Kopylov et al., 1987), their behaviour differs slightly: the radio jets deviate from the kinematics of the optical jets by more than $10 \%$ (e.g. Blundell \& Bowler, 2004; Schillemat et al., 2004; Roberts et al., 2008); the radio clouds can be found far away from the jets (e.g. Spencer, 1984; Romney et al., 1987); the radio jets appear to be more continuous (e.g. Roberts et al., 2008) vs. the bullet-like optical jets (Borisov \& Fabrika, 1987); and the radio jets undergo qualitative changes in a stream at distances of 0 '”050-0'.100 (the radio-brightening zone, Vermeulen et al., 1987 ) and $\sim 1^{\prime \prime} .5$ (the reheating zone, observed in the X-ray, Migliari et al., 2002). These peculiarities might be caused by the dynamics of the jets.

In the surroundings of SS 433, which are pervaded with the powerful wind of a mass flow-rate $\dot{M}_{\mathrm{w}} \sim 10^{-4} M_{\odot} / \mathrm{yr}$ and a velocity $v_{\mathrm{w}} \sim 1500 \mathrm{~km} \mathrm{~s}^{-1}$ from the supercritical accretion disk, the wind-jet ram pressure might radically influence the behaviour of the precessing jets, if not the wind anisotropy and evacuation of gas from the jet channels by the jets themselves. Nevertheless, 
the jets have to decelerate somewhere on the way to the shell of W 50: a flight-time $R_{\mathrm{W} 50} / v_{\mathrm{j}} \approx 80 \mathrm{pc} / 0.26 \mathrm{c} \sim 10^{3} \mathrm{yr}$ of the jet with a speed $v_{\mathrm{j}} \approx 0.26 c$ of the optical jets, where $c$ is the speed of light, over the W 50 largest radius $R_{\mathrm{W} 50}$ is much smaller than the age $\sim 10^{4}-10^{5}$ yr of W 50 (Begelman et al., 2006, and references therein). Moreover, it follows from the X-ray observations that the jets lose their helical appearance and are decelerated in the interior of W 50 (Brinkmann et al., 2007). At the same time, the jets are dark: only less than $1 \%$ of the huge kinetic luminosity $L_{\mathrm{k}} \sim 10^{39} \mathrm{erg} / \mathrm{s}$ of the jet is transformed into jet radiation and the heating of W 50, and the rest most likely transforms into mechanical energy of W 50, with an unbelievable effectiveness of $>99 \%$ (Dubner et al., 1998; Brinkmann et al., 2007).

Other indications of the deceleration are the following:

1. The difference in speed between the jets observed in the $\mathrm{X}$-ray and optical spectral domains $-0.2699 \pm 0.0007 c$ (Marshall et al., 2002) vs. $0.2581 \pm 0.0008 c$ (Davydov et al., 2008). This corresponds to a rate of loss of the jet kinetic energy $\dot{W}_{\mathrm{k}} / L_{\mathrm{k}} \approx 2 \delta v_{\mathrm{j}}=9 \%$ of the kinetic luminosity if the jet mass-flow rate is constant, where $\delta v_{\mathrm{j}}$ is the relative decrement of the speed.

2. As Kundt, (1987) has noted, the kinematic distance to the object determined by the kinematics of the extended jets (i.e. jets on a scale of several arcseconds) is larger than the distance determined by the kinematics of the inner jets (i.e. jets on a sub-arcsecond scale): $5.5 \pm 0.2 \mathrm{kpc}$ (Hjellming \& Johnston, 1981; Blundell \& Bowler, 2004) from images unresolved in time of the extended jets vs. the estimates $4.9 \pm 0.2 \mathrm{kpc}$ (Spencer, 1984), $4.85 \pm 0.2 \mathrm{kpc}$ (Vermeulen et al., 1993) and $4.61 \pm 0.35 \mathrm{kpc}$ (Stirling et al., 2002) from proper motion of radio knots in the inner jets, and $5.0 \pm 0.3 \mathrm{kpc}$ (Fejes, 1986) and 5.0 $\pm 0.5 \mathrm{kpc}$ (Romney et al., 1987) from images unresolved in time of the inner jets. This dichotomy might be explained by the fact that an a priori assumption of constancy of the jet velocity in kinematic simulations of really decelerating jets leads to an overestimate of the distance to SS 433, $D$, so that the model jet matches the observed angular jet size, which is proportional in the first approximation to the ratio $v_{\mathrm{j}} / D$. Roberts et al., (2008) noted that when they set the settings $v_{\mathrm{j}} / D=0.2647 \mathrm{c} / 5.5 \mathrm{kpc}$ for the extended jets, the kinematics of the inner jets agree better with the settings $0.2647 c / 5.0 \mathrm{kpc}$ or $0.29 c / 5.5 \mathrm{kpc}$. In this regard, they suggested variations of the radio jet velocity of about $\pm 10 \%$.

3. The velocity profile along the radio jets derived by Blundell \& Bowler, (2004, Fig. 4) for a particular image shows variations of $v_{\mathrm{j}}$ with a rate of the jet speed change $\leq 0.018 c / P_{\mathrm{pr}}$, where $P_{\mathrm{pr}}$ is the precession period, which might be a result of the uneven deceleration in the jets with the settings $0.29 \mathrm{c} / 5.5 \mathrm{kpc}$ adopted by Roberts et al., (2008) for the inner jets.

Stirling et al., (2004) have found that the fit to both the inner and extended radio jets on the same image is much better when the jet kinematic model, for the distance $D=4.8 \mathrm{kpc}$, sets the deceleration to $0.02 c / P_{\mathrm{pr}}$. Bell et al., (2011) have noticed that a satisfactory fit can be obtained for a constant speed when they used the larger distance $D=5.5 \mathrm{kpc}$. However, the latter choice does not eliminate the distance dichotomy resulting from many observations, and the necessity of introducing of deceleration into the standard kinematic model appears in every attempt to fit the radio jets on all scales simultaneously (Z. Paragi, 2009, priv. comm.).
Recently a brightness profile of the synchrotron radio emission along the jets of SS 433 up to the flight-times (or ages) of $\sim 800$ days was reported (Roberts et al., 2010; Bell et al., 2011). The relativistic electrons responsible for this emission are probably accelerated in the shock waves that result from the collision of the jets with the ambient medium (Heavens et al., 1990; Paragi et al., 2003). This profile allows one to evaluate the dynamic pressure of the medium on the jets and, as a result, their deceleration. In the present work, we used this profile to study the model of the radio jets that satisfies the observed kinematics of both the internal and the external jets at the same distance from the observer.

\section{Dynamics of SS 433 jets}

The morphology of the radio jets in the maps of Roberts et al., (2008) gives a clear indication of a significant role in its formation of the dynamic pressure of the surroundings that arises in the course of the jet precession. In addition, Roberts et al., (2010) argued that the form of the Doppler boosting of the observed intensities of the jets is very characteristic of a continuous jet (see also Stirling et al., 2004). However, the optical jets consist of clouds (e.g. Panferov \& Fabrika, 1997), and the milliarcsecond-scale radio jets are apparently knotty (e.g. Vermeulen et al., 1993), which, we note, does not exclude a continuous underlying stream. Miller-Jones et al., (2008) also argued for a cloudy structure of the external radio jets. Here, we propose a model in which the SS 433 jet is quasi-continuous and the ambient gas swept up by the jet provides dynamic pressure on the jet surface, which results in a deviation of the jet from ballistic kinematics. At the same time, the jet pattern speed $v_{\mathrm{n}} \simeq v_{\mathrm{j}}\left(1+\left(v_{\mathrm{j}} / v_{\phi}\right)^{2}\right)^{-0.5}$, where $v_{\phi}$ is the azimuthal velocity of the jet due to the precession and nutation, may be large enough to excite the shock waves on the surface, which spread inside the jet and cause strong turbulence around the clouds in the jet and, as a result, strengthen the magnetic field and very effectively accelerate relativistic particles, as was shown for supernova remnants by Inoue et al., (2012). Therefore, in contrast to the model of Hjellming \& Johnston, (1988), in our model the synchrotron emission occurs not on the surface of a homogeneous jet, but in the vicinity of the dense clouds, and the jet is not adiabatic, but is heating to temperatures $T \gtrsim 10^{7} \mathrm{~K}$ downward of the jet (Migliari et al., 2002).

According to this model, the jet average internal pressure $p_{\text {in }}$, equal to the sum of the pressures of the magnetic field $p_{\mathrm{m}}$, relativistic particles $p_{\mathrm{r}}$ and gas $p_{\mathrm{g}}$, is approximately equal the dynamic pressure $p_{\text {dyn }}$ on the surface of the jet, neglecting pressures of magnetic field and gas of the environment - we set their equality:

$p_{\text {dyn }}=p_{\text {in }} \equiv p_{\mathrm{m}}\left(1+\frac{1}{3 \beta_{\mathrm{H}}}+\beta_{\mathrm{g}}\right) \equiv p_{\mathrm{m}} \beta_{\mathrm{in}}$,

where $\beta_{\text {in }}=1+1 / 3 \beta_{\mathrm{H}}+\beta_{\mathrm{g}}, \beta_{\mathrm{H}}=\epsilon_{\mathrm{H}} / \epsilon_{\mathrm{r}}$ is the ratio of the energies of magnetic field, $\epsilon_{\mathrm{H}}$, and all relativistic particles, $\epsilon_{\mathrm{r}}, \beta_{\mathrm{g}}=p_{\mathrm{g}} / p_{\mathrm{m}}$. A profile of the magnetic pressure $p_{\mathrm{m}}$ along the jet is evaluated by using the rest-frame spectral density of synchrotron radiation flux $S_{v}$ (hereafter brightness), assuming a power-law energy spectrum of the electrons (e.g. Ginzburg, 1979, p. 97), as follows:

$p_{\mathrm{m}} \equiv \frac{H^{2}}{8 \pi}=\left(\frac{k_{v} \beta_{\mathrm{H}} \beta_{\mathrm{e}} D^{2} S_{v}}{V}\right)^{4 / 7}$, 


\section{A. A. Panferov (): Simulation of kinematics of SS 433 radio jets}

where $\beta_{\mathrm{e}}=\epsilon_{\mathrm{r}} / \epsilon_{\mathrm{e}}$ is the ratio of the energy of all relativistic particles to the energy $\epsilon_{\mathrm{e}}$ of relativistic electrons, $H$ is the strength of the magnetic field, which is assumed to be randomly directed, and $V$ is the volume of the radiating gas within the observing beam. The coefficient $k_{v}$ is $5.47 \times 10^{17}$ in the case of SS 433: the synchrotron spectral index is $\alpha=0.74\left(S_{v} \propto v^{-\alpha}\right)$, the frequency $v=4.86 \mathrm{GHz}$ of the brightness profile $S_{v}(r)$ from Bell et al., (2011), and the frequency range $4.86-\infty \mathrm{GHz}$ is accepted as the whole range of the synchrotron spectrum of the relativistic electrons. The pressure $p_{\mathrm{m}}$ may be underestimated by at most 2-3 times due to an uncertainty of the lower border of the synchrotron spectrum.

In our model, the magnetic field inside the jets and, therefore, the formation of the synchrotron radiation are localized in the vicinity of dense clouds, in the cloud shells, whose thickness $l_{\mathrm{sh}}$ is approximately equal to a half the size of the clouds, $l_{\mathrm{cl}}$, as Inoue et al., (2012) have demonstrated for clouds in supernova remnants. The volume of these cloud shells per jet segment of unit length is

$V=\frac{k_{\mathrm{V}}}{\rho_{\mathrm{cl}}} \frac{\dot{M}_{\mathrm{j}}}{\lambda_{\mathrm{j}}}=\frac{k_{\mathrm{V}} k_{\mathrm{B}} T_{\mathrm{cl}}}{\mu m_{\mathrm{p}} \beta_{\mathrm{in}} p_{\mathrm{m}}} \frac{\dot{M}_{\mathrm{j}}}{\lambda_{\mathrm{j}}}$,

where $k_{\mathrm{V}}$ is the volume ratio of the shells and clouds, $\rho_{\mathrm{cl}}=$ $\mu m_{\mathrm{p}} p_{\mathrm{cl}} / k_{\mathrm{B}} T_{\mathrm{cl}}$ and $T_{\mathrm{cl}}$ the mass density and temperature of the clouds, whose gas pressure $p_{\mathrm{cl}}$ is assumed to be equal to the jet average internal pressure $p_{\text {in }}=\beta_{\text {in }} p_{\text {m }}$ (Eq. (1)), $\dot{M}_{\mathrm{j}}$ is the flux of the jet mass contained in the clouds, $\lambda_{\mathrm{j}}$ is the jet length per unit of flight-time, $\mu$ is the average relative molar mass of the clouds ( $\approx 0.6$ for solar abundances), $m_{\mathrm{p}}$ is the proton mass, and $k_{\mathrm{B}}$ is the Boltzmann constant. In the adopted case of spherical clouds, $k_{\mathrm{V}}=\left(2 l_{\mathrm{sh}} / l_{\mathrm{cl}}+1\right)^{3}-1$. After substituting the volume (3) and the corresponding brightness per jet unit length, that is, the differential brightness $s_{v}=\mathrm{d} S_{v} / \mathrm{d} l$ which depends on the jet length $l$, into Eq. (2), the latter is transformed into

$p_{\mathrm{m}}=\left(\frac{k_{v} \beta_{\mathrm{in}} \beta_{\mathrm{H}} \beta_{\mathrm{e}} \mu m_{\mathrm{p}} \lambda_{\mathrm{j}} D^{2} s_{v}}{k_{\mathrm{V}} k_{\mathrm{B}} \dot{M}_{\mathrm{j}} T_{\mathrm{cl}}}\right)^{4 / 3}$

The temperature of the clouds in the SS 433 radio jets presumably increases downward of the jets, as suggested by the observed X-ray brightening of the jets (Migliari et al., 2002). In our model, the power-law profile $T_{\mathrm{cl}}(r)=T_{0} r^{\mathrm{n}}$ was adopted. Given the profiles of pressure and temperature, the distance of validity of the model of the cloud thermodynamics is restricted by the following condition on the volume filling of the jet by the clouds:

$$
f(r)=\frac{\dot{M}_{\mathrm{j}}}{\rho_{\mathrm{cl}}} \frac{1}{V_{1}}=\frac{k_{\mathrm{B}} T_{\mathrm{cl}} \dot{M}_{\mathrm{j}}}{\mu m_{\mathrm{p}} \beta_{\mathrm{in}} p_{\mathrm{m}}} \frac{1}{\pi\left(r \theta_{\mathrm{jr}} / 2\right)^{2} v_{\mathrm{j}}}<1 .
$$

Here, a conical geometry of the jet is assumed, with the opening $\theta_{\mathrm{jr}}$, and the jet volume $V_{1}$ per unit of flight-time is approximated by the cylinder volume $\pi\left(r \theta_{\mathrm{jr}} / 2\right)^{2} v_{\mathrm{j}}$, because the expansion of the jet is negligible: $v_{\mathrm{j}} / r \ll 1$.

The extended radio jets do not show a helical pattern of the nutation, although there are signs of the nutation of the radio jets within the brightening zone (Vermeulen et al., 1993; Mioduszewski \& Rupen, 2007). In the inner jets, the magnetic field is ordered and aligned with the jet precession helix (Roberts et al., 2008). Nutation would smear such order. All this suggests that the nutation structure of the radio jets blurs with the distance $r$, and the jets acquire the opening $\theta_{\mathrm{jr}}=\theta_{\mathrm{j}}+\theta_{\mathrm{n}}=6: 8$, where $\theta_{\mathrm{j}}=1.2$ and $\theta_{\mathrm{n}}=5: 6$ are the opening and the nutation cone angle of the optical jets, respectively (Borisov \& Fabrika, 1987). In this case, the angular velocity of the radio jets is determined only by the precession, $\omega=2 \pi / P_{\mathrm{pr}}$, and the azimuthal velocity is $v_{\phi}=\omega r \sin \theta_{\mathrm{pr}}$. The length $\lambda_{\mathrm{j}}=\left|\mathbf{v}_{\mathrm{j}}(r)-\mathbf{v}_{\phi}(r)\right|$ is determined by the vectors $\mathbf{v}_{\mathrm{j}}(r)$ and $\mathbf{v}_{\phi}(r)$ of the jet radial and azimuthal velocities, respectively. In general, while the distance $r$ increases, $\mathbf{v}_{\mathrm{j}}(r)$ deviates from the ejection direction hereafter the longitudinal $Z$-axis of the Cartesian rest-frame of reference of the ballistic jet, with the azimuthal $Y$-axis chosen to be directed oppositely to the precession movement, that is to $\mathbf{v}_{\phi}(r)$, which is counterclockwise, viewed from the jet source (see Stirling et al., 2002, for a scheme of the jet precession and Fig. 1 for this frame of reference).

The rest-frame brightness profile of the radio jet is approximated as

$S_{v}(r)=S_{0} \exp \left(-\frac{r}{\tau}\right)$

with the brightness $S_{v}=32.7 \mathrm{mJy}$ per beam of the telescope of a size $\phi_{\mathrm{b}}=0,32$ at $v=4.86 \mathrm{GHz}$ at the distance $r=50^{\mathrm{d}} \cdot v_{\mathrm{j}}^{*}$, and the decrement $\tau=55 \mathrm{~d} 9 \cdot v_{\mathrm{j}}^{*}$ at the flight-times $t=50$ $250^{\mathrm{d}}$, where $v_{\mathrm{j}}^{*}=0.2647 \mathrm{c}$ is the fiducial speed (Roberts et al., 2010; Bell et al., 2011). Given expression (6) for the brightness, the differential brightness will also be the exponential function $s_{v}(r)=s_{0} \exp (-r / \tau)$, with the normalization factor $s_{0}=S_{0} / 2 \tau \sinh \left(l_{\mathrm{b}} / 2 \tau\right)$ determined by Eq. (6) and the normalization condition

$$
S_{v}(r)=\int_{r-l_{\mathrm{b}} / 2}^{r+l_{\mathrm{b}} / 2} s_{v}(\zeta) \mathrm{d} \zeta=s_{0} \tau\left(\exp \left(\frac{l_{\mathrm{b}}}{2 \tau}\right)-\exp \left(-\frac{l_{\mathrm{b}}}{2 \tau}\right)\right) \exp \left(-\frac{r}{\tau}\right)
$$

where $l_{\mathrm{b}}=\phi_{\mathrm{b}} D$ is the linear size of the beam. The differential brightness $s_{v}(r)$ grows exponentially upward of the jet at least to flight-times $\sim 10^{\mathrm{d}}$ (Roberts et al., 2008). For shorter times, we extrapolated $s_{v}(r)$. The differential brightness in dependency on the jet length is obtained from the condition of conservation of $s_{v}(r) \mathrm{d} r$ when replacing the independent variable $r$ by $l$, as $s_{v}(l)=s_{v}(r) v_{\mathrm{jz}}(r) / \lambda_{\mathrm{j}}(r)$, where we replaced $v_{\mathrm{j}}=\mathrm{d} r / \mathrm{d} t=v_{\mathrm{jx}} x / r+v_{\mathrm{jy}} y / r+v_{\mathrm{j} z} z / r$ by the $z$-component of the jet velocity $v_{\mathrm{jz}}(r)$ in the coordinate system defined above, but with the axes origin at the source, because $x, y \ll z$ and $v_{\mathrm{jx}}, v_{\mathrm{jy}} \ll v_{\mathrm{jz}}$. In the case of ballistic jets, we note that $x, y, v_{\mathrm{jx}}, v_{\mathrm{jy}}=0$. The transverse size of the jet projection onto the plane of the sky is smaller than the size of the beam, and, hence, the definition of the differential brightness $s_{v}(r)$ of the jet is valid at the flighttimes $<\phi_{\mathrm{b}} D / \theta_{\mathrm{jr}} v_{\mathrm{j}} \approx 290^{\mathrm{d}}$.

Now we have all constituents to write the equation of the dynamics

$\mathbf{a}=-\eta \frac{p_{\mathrm{m}} \beta_{\mathrm{in}} \lambda_{\mathrm{j}} r \theta_{\mathrm{jr}}}{\dot{M}_{\mathrm{j}}} \mathbf{e}_{\mathrm{n}}$

of a jet segment, independent of other segments, of the length $\lambda_{\mathrm{j}}$, of the transverse size $r \theta_{\mathrm{jr}}$, of the mass $\dot{M}_{\mathrm{j}}$, which is under the dynamic pressure $p_{\text {dyn }}=\beta_{\text {in }} p_{\mathrm{m}}$ (Eq. (1)). Here, $\eta \lesssim 1$ is the geometrical factor that characterizes the streamlining of the jet by swept-up gas and depending on the transverse profile of the jet, and $\mathbf{e}_{\mathrm{n}}$ is the unit vector of velocity of the jet pattern relative to the ambient medium, which presumably is at rest. Equation (8), in fact an equation of the dynamics of the material point, 


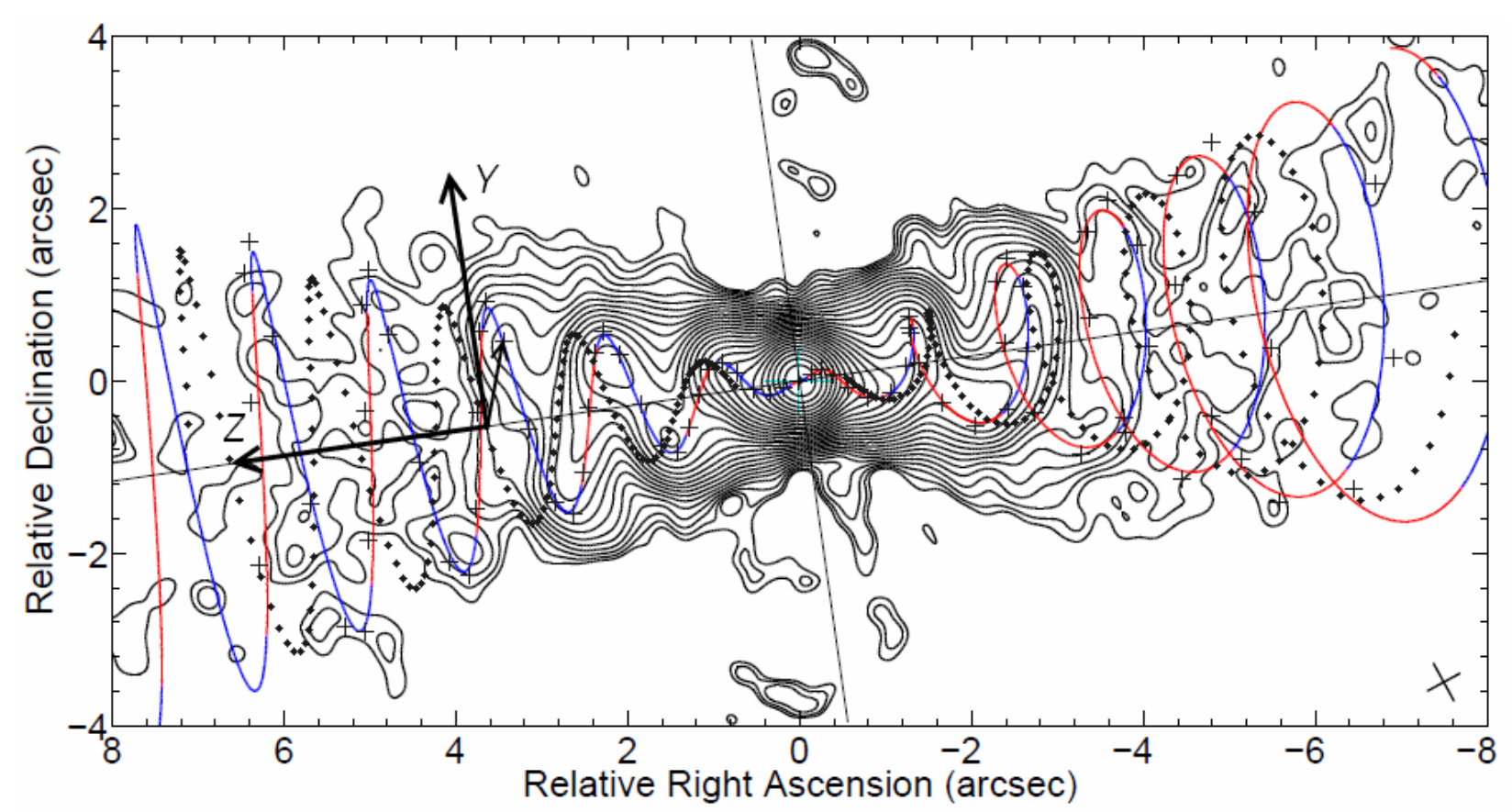

Fig. 1. Simulated loci of the radio jets of SS 433 for the settings $v_{\mathrm{j}} / D=0.2581 \mathrm{c} / 4.8 \mathrm{kpc}-$ the dynamic jet loci are marked by pluses every $20^{\mathrm{d}}$ of a flight, and the ballistic jet loci are marked by dots every $5^{\mathrm{d}}$ beginning from a flight-time of $P_{\mathrm{pr}} / 2-$ are superimposed on the image of the jets (courtesy of Dr. D.H. Roberts). Every simulated jet trace has a length of 800 flight days. The solid helical line is the ballistic jet loci drawn by Roberts et al., (2010) for the settings $v_{\mathrm{j}} / D=0.2647 c / 5.5 \mathrm{kpc}$. Approaching/receding parts of this helix are blue/red (in the on-line version of this paper). We show the longitudinal $Z$ and azimuthal $Y$ axes of the rest frame of reference of the ballistic jet of our simulation, with the axes origin located at a particular jet segment. The displacement vector between this segment and the segment of the same flight-time in the dynamic jet demonstrates the deviation of the kinematics of the dynamic jets from the ballistic kinematics.

correctly describes the behaviour of a quasi-continuous precessing jet, while the displacement of the jet from the ballistic (unaccelerated) position is relatively small.

At angular distances beyond $\sim 4^{\prime \prime}$, or $t \sim 430^{\mathrm{d}}$, the SS 433 radio jets look fragmentary (e.g. Roberts et al., 2010, Fig. 2) likely the jets probably do not constitute a continuous stream and our model is not valid there.

\section{Simulation of the dynamical jets of SS 433}

The acceleration (8) was used to simulate the locus of the SS 433 jets. The initial conditions of this kinematic problem are defined by the standard kinematic model with the most accurate parameters to date collected in Table 1. A distance to SS 433 of $4.8 \mathrm{kpc}$ was adopted, which is thought to be minimally biased by the acceleration (see above). This distance is possibly even smaller. The simulated locus was projected onto the plane of sky, accounting for the time delay of arriving photons to the observer that distorts image of the jets (the light travel effect).

The model jet loci are shown in Fig. 1 overplotted on the deep image of the radio jets of SS 433 on July 11, 2003 from Roberts et al., (2010), where the jets are seen up to $\sim 800$ days of flight. The dynamic jets simulated by our model with the settings $v_{\mathrm{j}} / D=0.2581 \mathrm{c} / 4.8 \mathrm{kpc}$ for the initial speed and distance, and the ballistic jets obtained by Roberts et al., (2010) for the settings $v_{\mathrm{j}} / D=0.2647 c / 5.5 \mathrm{kpc}$ that were used by us as a template for the comparison, fit the observed radio jets equally well: these two model loci are visually almost indistinguishable in both the inner and external parts, the difference between them at a length $3^{\prime \prime}$ is lower than the accuracy of 0 '.035 of the determination of the jet ridge in this image reported by Blundell \& Bowler, (2004).
Table 1. Parameters of the standard kinematic model of the SS 433 jets.

\begin{tabular}{lcc}
\hline \hline Kinematic parameter & Symbol and value & Ref. \\
\hline Jet ejection speed & $v_{\mathrm{j}}=0.2581 c$ & 1 \\
Precession axis inclination & $i=78.81$ & 1 \\
Precession axis position & $\chi=98.2$ & 2 \\
Precession cone half-angle & $\theta_{\mathrm{pr}}=19 \circ 75$ & 1 \\
Precession period & $P_{\mathrm{pr}}=162.250 \mathrm{~d}$ & 1 \\
Initial phase epoch $^{a}$ & $t_{0}=\mathrm{JD} 2443508.41$ & 1 \\
\hline
\end{tabular}

Notes. ${ }^{(a)}$ When the east jet is closest to the line of sight.

References. (1) Davydov et al., (2008); (2) Stirling et al., (2002).

For the settings of the jet dynamics we chose the following characteristic values: a geometry factor $\eta=1$; a ratio $l_{\mathrm{sh}} / l_{\mathrm{cl}}=0.5$ of the thickness of the radio bright shells around the jet clouds to the size of the clouds, the finding of Inoue et al., (2012) for supernova remnants; a pressure ratio of the thermal gas and magnetic field $\beta_{\mathrm{g}}=1$, which is expected in the vicinity of the jet clouds, where the magnetic field is amplified by the shocks (Jones et al., 1996; Inoue et al., 2012); a ratio of the energy densities of the magnetic field and relativistic particles $\beta_{\mathrm{H}}=3 / 4$, which corresponds minimum total energy of the field and particles (Ginzburg, 1979, p. 95); an initial flux of kinetic energy in the jet per cloud fraction, $L_{\mathrm{k}} \equiv \dot{M}_{\mathrm{j}} v_{\mathrm{j}}^{2} / 2=10^{39} \mathrm{erg} / \mathrm{s}$, and a temperature of the jet clouds $T_{\mathrm{cl}}=2 \times 10^{4} \mathrm{~K}$ at a distance of $10^{15} \mathrm{~cm}$, which are very tightly determined for the optical jet (Panferov \& Fabrika, 1997). The jet mass-flux $\dot{M}_{\mathrm{j}}$ was assumed to be constant throughout the jet length. Only two parameters were fitted: an exponent of the cloud temperature profile $n=1.50_{-0.05}^{+0.07}$, which tunes the equality of goodness of the fits 
Table 2. Dynamical parameters of the SS 433 jets.

\begin{tabular}{lc}
\hline \hline Parameter & Value \\
\hline Shell/cloud thickness ratio, $l_{\mathrm{sh}} / l_{\mathrm{cl}}$ & 0.5 \\
Gas/magnetic pressure ratio, $\beta_{\mathrm{g}}$ & 1 \\
Magnetic/particle energy ratio, $\beta_{\mathrm{H}}$ & $3 / 4$ \\
Relativistic particle/electron energy ratio, $\beta_{\mathrm{e}}$ & $2.7 \pm 0.4$ \\
Jet initial kinetic luminosity, $L_{\mathrm{k}}$ & $10^{39} \mathrm{erg} / \mathrm{s}$ \\
Cloud temperature, $T_{\mathrm{cl}}\left(10^{15} \mathrm{~cm}\right)$ & $2 \times 10^{4} \mathrm{~K}$ \\
Cloud temperature exponent, $n$ & $1.50_{-0.05}^{+0.07}$ \\
\hline
\end{tabular}

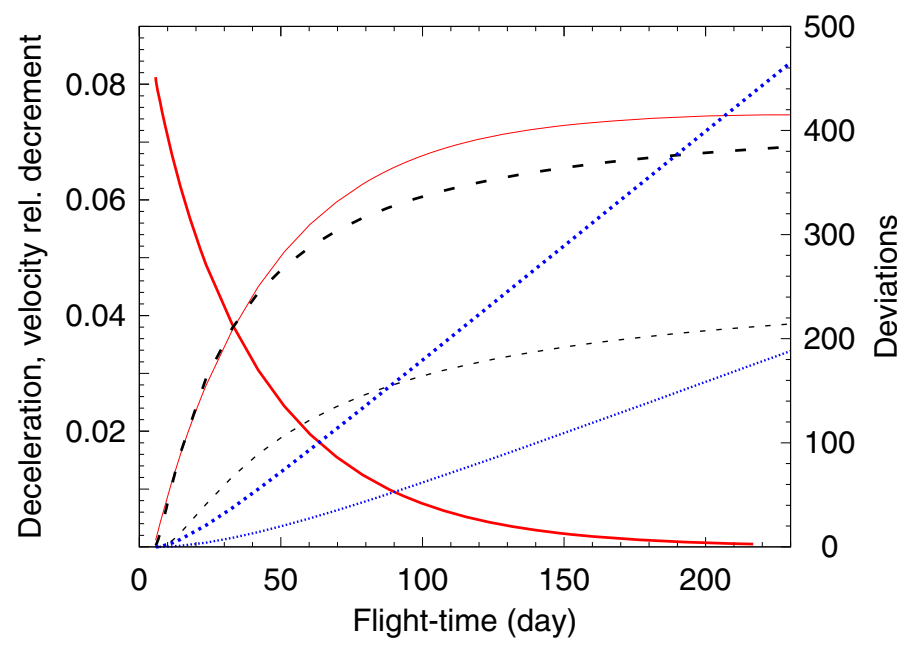

Fig. 2. Deviations of kinematical parameters of the dynamical jets from those of the ballistic jets in dependency on the flight-time. Along the left axis, thick and thin solid red lines: tangential deceleration $a_{\tau}(t)$, in units of $c / P_{\mathrm{pr}}$, and relative decrement $\delta v_{\mathrm{j}}(t)$ of the jet velocity, respectively. Along the right axis, thick and thin dotted blue lines: transverse $\Delta y(t)$ and longitudinal $-\Delta z(t)$ deviations, respectively, of the dynamical jet loci in the comoving frame of reference of the ballistic jet, in units of mas; thick and thin dashed black lines: increment of azimuth $\Delta \psi(t)$ of the precession rotation, in units of a tenth of a degree, and increment $\Delta \theta_{\mathrm{pr}}(t)$ of the precession cone semi-opening, in units of a hundredth of a degree, respectively. This figure is available in colour in the on line version of this paper.

to the inner and extended jets, and a ratio of the energies of all the relativistic particles and relativistic electrons $\beta_{\mathrm{e}}=2.7 \pm 0.4$, although this is not an independent fitting parameter. The accuracies of the fitted parameters correspond to a deviation of the dynamical jets from the template in Fig. 1 of half the image resolution 0 '.47 at distances of about $6^{\prime \prime}$, where the helix of the eastern jet is still clearly discernible. These best dynamical parameters are summarized in Table 2. In the simulations, the jet dynamics was taken into account only in the region from the radio-brightening zone to a distance $t_{1}=215^{\mathrm{d}}$ of flight where the filling factor $f$ tends to 1 and, as a result, the model of the jet dynamics becomes invalid.

A deviation of the kinematics of the dynamic jets with the parameters given above from the kinematics of the ballistic jets, with the same settings $v_{\mathrm{j}} / D=0.2581 \mathrm{c} / 4.8 \mathrm{kpc}$, can be seen in Fig. 1 and is characterized in Fig. 2 by differences of the following kinematic parameters in dependency on flight-time: tangential acceleration, velocity, coordinates $y$ and $z$ in the frame of reference comoving with the ballistic jet, azimuthal angle of the precession $\psi$, and precession cone half-angle. The profile of deceleration $a_{\tau}(t) \equiv-\dot{v}_{\mathrm{j}}(t)$, which is the length of the tangential component of the jet acceleration vector (8), shows a decrease of the deceleration downward of the jets from a peak value of $0.081 c / P_{\mathrm{pr}}$ in the radio-brightening zone, as Stirling et al., (2004) suggested. This means that the deceleration takes place mainly within the inner jets. An average value of this deceleration in the first precession cycle is $0.019 c / P_{\mathrm{pr}}$. A full relative decrement of the jet speed is $\delta v_{\mathrm{j}} \equiv 1-v_{\mathrm{j}}\left(t_{1}\right) / v_{\mathrm{j}}(0)=7.5 \%$, half of this is accumulated at a distance of $t_{1 / 2}=33^{\mathrm{d}}$, that is, during one-fifth of the precession period. Approximately the same distance confines the zone of significant slowdown of the jets: the jet-medium interaction beyond this distance leads to a shift of the precession spiral at distances of about 6" no more than half of the image resolution. Therefore, at $t>P_{\mathrm{pr}}$ the external jets are expected be significantly ballistic. During a flight-time of $P_{\mathrm{pr}}$, or in bounds of 1'.389 from the jet source, the precession cone semiopening increases by $\Delta \theta_{\mathrm{pr}}=2: 0$, and the jet azimuth increases by $\Delta \psi=37^{\circ}$ (that is, the precession phase $1-\psi / 2 \pi$ decreases by 0.10 ), or the jets become offset along the azimuth direction by $\Delta y=316$ milliarcsec (mas), which is approximately 2.6 times more than the longitudinal offset $|\Delta z|=121$ mas against the $Z$-axis (note that the distance $r$ decreases by the lower value $|\Delta r|=r-\sqrt{(r-|\Delta z|)^{2}+\Delta y^{2}}=85$ mas $)$ - the precession helix shrinks along the precession axis and curls even more. This is expected to be detected as a slowing-down and precession lag of the observed jets compared with the standard kinematic model, although the curling is visually perceived merely as the shrinking and therefore cannot be distinguished in the jet observations that not resolved in time. The average visual slowdown of the dynamical jets, which is related to the distances difference $\Delta r_{\text {vis }}$ between the segments of ballistic and dynamic jets of the same azimuth, is $0.077 c / P_{\mathrm{pr}}$ during the first precession cycle and $0.027 c / P_{\mathrm{pr}}$ during a flight-time of $350^{\mathrm{d}}$. The latter is higher than the value $0.02 c / P_{\mathrm{pr}}$ found by Stirling et al., (2004) for approximately the same flight-time. These authors also took note of the delay of the jets in precession phase, by 0.25 . A full visual relative decrement of the jet velocity is $\delta v_{\mathrm{j} \text { vis }}=\Delta r_{\mathrm{vis}}\left(t_{1}\right) / t_{1} v_{\mathrm{j}}(0)=13 \%$, neglecting the deceleration.

\section{Discussion}

We found that the SS 433 radio jets must be under a considerable dynamical impact, if relativistic electrons responsible for the observed radiation are steadily injected by the shocks inside the jet, which are triggered by the shocks excited at the jet surface. In the collision with the ambient medium the jet acquires additional momentum whose vector lies in the tangential plane $(Y Z)$ to the precession cone and causes the jet to decelerate along the $Z$-axis and shift along the $Y$-axis. The acquired shift, demonstrated in Fig. 1 by the displacement vector, must be observed as an increase of torsion of the precession helix, or as a decrease of the precession phase. This might be the reason why the precession phase of every radio jet observation deviates from the kinematic model (Z. Paragi, 2009, priv. comm.). Note that the known azimuthal asymmetry of radiation of the optical jets points to a similar character of the jet-medium interaction (Panferov et al., 1997). If the collision occured at the front of the jet clouds, along the $Z$-axis, one would expect only a deceleration of the radio jets - in this case, the dynamical model fits the observed jets for no values of the dynamical parameters. The significant offset $\Delta y$ of the dynamical jets allows us to explain the appearance of individual clouds outside of the SS 433 radio jet trace as a result of the uneven ram pressure of the inhomogeneous wind on the fragmented jets.

The dynamical model fits the radio jets of SS 433 well when the object is placed at a distance of $4.8 \mathrm{kpc}$. The deceleration is 
highest in the radio-brightening zone and decreases downward the jet. Accordingly, the strongest radio jet radiation is associated with the highest jet kinetic energy loss. The zone of the (significant) deceleration spans a flight-time of only one-fifth of the precession period. Beyond the deceleration zone the dynamical jets imitate ballistic jets with a speed $0.2647 c$ and at the larger distance to the object $5.5 \mathrm{kpc}$. There are contradictory estimates of the distance (see Lockman et al., 2007, and references therein), including those obtained by the kinematic method on the basis of observations of the inner and extended radio jets. The dynamical model solves the dichotomy of the kinematic distance.

Blundell \& Bowler, (2004) have fitted the extended radio jets by the ballistic model by allowing the jet speed to vary stochastically. The speed variation magnitude they found is $0.032 c(4.8 \mathrm{kpc} / 5.5 \mathrm{kpc})=0.028 c$, or $\approx 11 \%$ of the jet speed, within the parameters of our model, which is comparable with the visual relative decrement $\delta v_{\mathrm{j} \text { vis }}=13 \%$ issued from the jet dynamics. The observed speed variations may be partly due to uneven deceleration in the inner jets ${ }^{2}$. As for the distance to SS 433, the results of Blundell \& Bowler, (2004) for the extended radio jets do not depend on the deceleration, which occures in the inner jets, nor exclude the dynamic model, which shows that the extended jets are ballistic with the parameters $v_{\mathrm{j}}=0.2387 c$ and $D=4.8 \mathrm{kpc}$, which are intermediate to the two options they have discerned on the basis of the light travel effect.

It is quite possible that the blurring of the nutation structure of the jets occures within the deceleration zone, because the ram pressure on the jets, depending on the jet azimuthal velocity, is strongly modulated with nutation phase. In this case the jet velocity variations normal to the jet axis have to be of the order of $z \theta_{\mathrm{n}} v_{\mathrm{j}} \approx 0.1 v_{\mathrm{j}}$ - exactly the magnitude of the observed jet speed variations during the first 40 flight days (Schillemat et al., 2004). In addition, the blurring may be initiated by abrupt heat and expansion of the jet clouds and decollimation of the jets in the radio-brightening zone, where pressure in the jets probably decreases steeply (Vermeulen et al., 1993; Panferov, 1999). The lack of the nutation structure in the radio jets may also be a manifestation of the two-stream structure of the jets in the beginning: with the nutating central stream, observed in the optics, and the wider shell stream, which damps the nutation. Jets with this structure might arise in scenarios of the type described by Begelman et al., (2006).

The huge power loss of kinetic energy of the radio jet $\dot{W}_{\mathrm{k}} \approx$ $2 \delta v_{\mathrm{j}} L_{\mathrm{k}}=1.50 \times 10^{38} \mathrm{erg} / \mathrm{s}$ is not observed radiatively. Possibly, this power is drained into mechanical energy of the wind that interacts with the jets, and is eventually transformed into mechanical energy of the W 50 shell (Dubner et al., 1998). Similarly, the high efficiency of the mechanical energy transfer from jets to atomic and molecular outflows in the surroundings is observed in AGNs (Morganti et al., 2013).

The model of the jet dynamics unites different properties of the SS 433 radio jets such as the dynamics and radiation. The parameters of this model are not independent of each other and may be subjected to transformations that do not change the found profile of the jet acceleration, by the following formula:

$a(r)=k_{\mathrm{a}}(r)\left(\frac{\beta_{\mathrm{in}}}{\dot{M}_{\mathrm{j}}}\right)^{7 / 3}\left(\frac{\beta_{\mathrm{H}} \beta_{\mathrm{e}}}{k_{\mathrm{V}} T_{0}}\right)^{4 / 3}$,

\footnotetext{
2 We also also note that in a patchy jet with an openning $\theta_{\mathrm{jr}}=6.8^{\circ}$, inclined to the line of sight by $\theta \geq \theta_{\mathrm{i}}=i-\theta_{\mathrm{pr}} \approx 59^{\circ}$, a natural spread of the apparent velocities of the patches $\Delta v_{\tau}=\Delta\left(v_{\mathrm{j}} \sin \theta\right)$ is on the order of $\Delta v_{\tau} / v_{\tau} \approx \Delta \theta \cot \theta \leq \theta_{\mathrm{jr}} \cot \theta_{\mathrm{i}}=7.1 \%$.
}

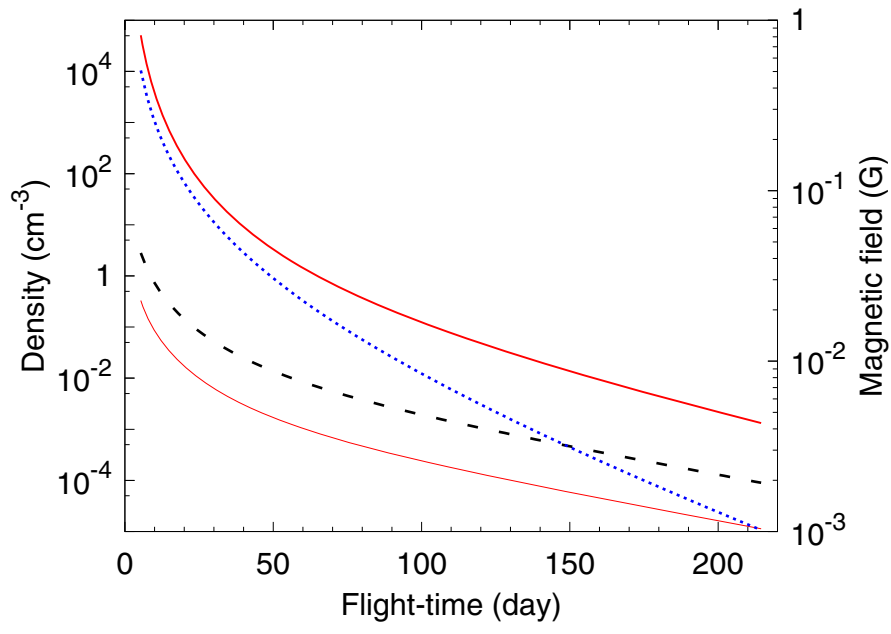

Fig. 3. Density of the ambient medium of the SS 433 jets (thick red line) and its ratios to the isotropic wind density (thin red line) and to the jets average density (dashed black line) are plotted along the left axis in dependency on flight-time. The strength of the magnetic field in the vicinity of the jet clouds (dotted blue line) is plotted along the right axis. This figure is available in colour in the online version of this paper.

obtained from (4) and (8), where $k_{\mathrm{a}}(r)=\eta r^{1-4 n / 3} \theta_{\mathrm{jr}} \lambda_{\mathrm{j}}^{7 / 3}$ $\left(k_{v} \mu m_{\mathrm{p}} D^{2} s_{v} / k_{\mathrm{B}}\right)^{4 / 3}$. The values of $l_{\mathrm{sh}} / l_{\mathrm{cl}}, \beta_{\mathrm{g}}, \beta_{\mathrm{H}}, \beta_{\mathrm{e}}, L_{\mathrm{k}}$ and $T_{0}$, given in Table 2 do not only provide the best fit of the dynamical model, but are also justified observationally and theoretically, but may be $\beta_{\mathrm{e}}$, which in turn validates the model. If we were to change the found suspiciously low value $\beta_{\mathrm{e}}=2.7$ by 100 , the value observed near Earth (Ginzburg, 1979, p. 96), then Eq. (9), with the invariant $a(r)$, dictates the change of $L_{\mathrm{k}}=1 \times 10^{39} \mathrm{erg} / \mathrm{s}$ by $7.9 \times 10^{39} \mathrm{erg} / \mathrm{s}$, or $l_{\mathrm{sh}} / l_{\mathrm{cl}}=0.5$ by 2.7 , or $T_{\mathrm{cl}}\left(10^{15} \mathrm{~cm}\right)=$ $2 \times 10^{4} \mathrm{~K}$ by $7.4 \times 10^{5} \mathrm{~K}-$ all of these are beyond the reasonable ranges. Similarly, the scope of the variations allowed by Eq. (9) of another dynamical parameter is severely restricted. There is a reserve for increase of $\beta_{\mathrm{e}}$ in the uncertainties of the parameters incorporated in $k_{\mathrm{a}}(r)$ : lowering of $\eta$ from the accepted unlikely maximum (see Table 2), $s_{v}$ by the off-jet fraction of the emission (Roberts et al., 2008), and $k_{v}$ due to the polarization of the emission, in which case the magnetic field averaged over all directions is expected to be weaker than in the case of the random field assumed in Eq. (2), all together could boost $\beta_{\mathrm{e}}$ by about a factor of 2 .

The density of the ambient medium, extracted from the dynamical model simulation by the formula $n_{\mathrm{a}}=$ $\beta_{\text {in }} p_{\mathrm{m}} / \eta m_{\mathrm{p}}\left(\mathbf{e}_{\mathrm{n}} \mathbf{v}_{\mathrm{j}}\right)^{2}$, is shown in Fig. 3. This density is essentially lower than the density $n_{\text {iso }} \propto \dot{M}_{\mathrm{w}} / v_{\mathrm{w}} r^{2}$ of the wind from the system's accretion disk by several orders of value, if this wind is isotropic - the jets propagate in almost empty channels in the wind. The exponent of the power-law fit $n_{\mathrm{a}} \propto t^{-m}$ to the profile in Fig. 3, ranging from $\sim 4$ to $\sim 6$ with flight-time, also shows the anisotropy of the wind. It also shows the density ratio of the ambient medium to the jets - it is essentially lower than 1 beyond the deceleration zone. The magnetic field in the vicinity of the jet clouds is shown in Fig. 3, conforming to the ram pressure by Eqs. (1), (2). In the radio-brightening zone $(t=5 \mathrm{~d} 4)$, the magnetic field strength is $0.51 \mathrm{G}$ which differs from the $0.08 \mathrm{G}$ found by Vermeulen et al., (1987) because we accounted for the clumping of the jets.

The volume-filling of the jets by the clouds increases with distance because the clouds are heated and expand as a result, while the cloud pressure, which is in balance with the jet internal pressure, is held by the ram pressure on the jets, which 


\section{A. A. Panferov (): Simulation of kinematics of SS 433 radio jets}

is falling. The cloud temperature rises from $1.4 \times 10^{5} \mathrm{~K}$ in the brightening zone to $3.0 \times 10^{7} \mathrm{~K}$ at the flight-times $\sim t_{1}=215^{\mathrm{d}}$ where the filling $f \rightarrow 1$ and the model becomes invalid. In fact, the jet kinematics is hardly sensitive to the dynamics prescribed by the model beyond the deceleration zone. Therefore, the cloud behaviour there, in particular the reheating, may be nonmonotonic without violating our findings on the jet kinematics. Given the temperature increment $\Delta T_{\mathrm{cl}}$, the power of jet heating is $k_{\mathrm{B}} \Delta T_{\mathrm{cl}}\left(3 \dot{M}_{\mathrm{j}} / 2 \mu m_{\mathrm{p}}\right)=2.1 \times 10^{35} \mathrm{erg} / \mathrm{s}$, which is quite enough to provide the observed jet X-ray luminosity $\sim 10^{33} \mathrm{erg} / \mathrm{s}$ and is surprisingly close to the radio jet synchrotron luminosity. All of this energy budget is minuscule in comparison with the powerloss of the jet kinetic energy $1.50 \times 10^{38} \mathrm{erg} / \mathrm{s}-$ we are left with the puzzle where the latter is deposited.

Our simulation shows that the radio jets of SS 433 can be decelerating and twisting in addition to the precession twist. In this case, the arcsecond-scale jets imitate ballistic jets at an inappropriate distance from the observer. An explicit detection of the jet dynamics is a quest for future observations of the inner radio jets.

\section{References}

Abell, G. O., \& Margon, B. 1979, Nature, 279, 701

Begelman, M. C., King, A. R., \& Pringle, J. E. 2006, MNRAS, 370, 399

Bell, M. R., Roberts, D. H., \& Wardle J. F. C. 2011, ApJ, 736, 118

Blundell, K. M., \& Bowler, M. G. 2004, ApJ, 616, L159

Borisov, N. V., \& Fabrika, S. N. 1987, Pis'ma v Astron. Zh., 13, 487

Brinkmann, W., Pratt, G. W., Rohr, S., Kawai, N., \& Burwitz, V. 2007, A\&A, 463, 611

Davydov, V. V., Esipov, V. F., \& Cherepashchuk, A. M. 2008, Astron. Rep., 52, 487

Dubner, G. M., Holdaway, M., Goss, W. M., \& Mirabel, I. F. 1998, ApJ, 116, 1842
Fabrika, S. 2004, Astrophys. Space Phys. Rev., 12, 1

Fejes, I. 1986, A\&A, 168, 69

Ginzburg, V. L. 1979, Theoretical Physics and Astrophysics (Oxford: Pergamon Press)

Heavens, A. F., Ballard, K. R., \& Kirk, J. G. 1990, MNRAS, 244, 474

Hjellming, R. M., \& Johnston, K. J. 1981, ApJ, 246, L141

Hjellming, R. M., \& Johnston, K. J. 1988, ApJ, 328, 600

Inoue, T., Yamazaki, R., Inutsuka, S.-I., \& Fukui, Y. 2012, ApJ, 744, 71

Jones, T. W., Ryu, D., \& Tregillis, I. L. 1996, ApJ, 473, 365

Kopylov, I. M., Kumaigorodskaja, R. N., \& Somov, N. N. 1987, AZh, 64, 785

Kundt, W. 1987, Ap\&SS, 134, 407

Lockman, F. J., Blundell, K. M., \& Goss, W. M. 2007, MNRAS, 381, 881

Marshall, H. L., Canizares, C. R., \& Schulz, N. S. 2002, ApJ, 564, 941

Migliari, S., Fender, R., \& Méndez, M. 2002, Science, 297, 1673

Miller-Jones, J. C. A., Migliari, S., Fender R. P., et al. 2008, ApJ, 682, 1141

Mioduszewski, A. J., \& Rupen, M. P. 2007, BAAS, 38, 954

Morganti, R., Frieswijk, W., Oonk, R. J. B., Oosterloo, T., \& Tadhunter, C. 2013, A\&A, 552, L4

Newsom, G. H., \& Collins, G. W., II. 1981, AJ, 86, 1250

Panferov, A. A. 1999, A\&A, 351, 156

Panferov, A. A., \& Fabrika, S. N. 1997, Astron. Rep., 41, 506

Panferov, A. A., Fabrika, S. N., \& Rakhimov, V. Yu. 1997, Astron. Rep., 41, 342

Paragi, Z., Stirling, A. M., \& Fejes, I. 2003, in 4th Microquasars Workshop, New Views on Microquasars, eds. Ph. Durouchoux, Y. Fuchs, \& J. Rodriguez (Kolkata, India: Center for Space Physics), 288

Roberts, D. H., Wardle, J. F. C., Lipnick, S. L., Selesnick, P. L., \& Slutsky, S. 2008, ApJ, 676, 584

Roberts, D. H., Wardle, J. F. C., Bell, M. R., et al. 2010, ApJ, 719, 1918

Romney, J. D., Schilizzi, R. T., Fejes, I., \& Spencer, R. E. 1987, ApJ, 321, 822

Schillemat, K., Mioduszewski, A., Dhawan, V., \& Rupen, M. 2004, BAAS, 36, 1515

Spencer, R. E. 1984, MNRAS, 209, 869

Stirling, A. M., Jowett, F. H., Spencer, R. E., Paragi, Z., \& Ogley, R. N. 2002, MNRAS, 337, 657

Stirling, A. M., Spencer, R. E., Cawthorne, T. V., \& Paragi, Z. 2004, MNRAS, 354,1239

Vermeulen, R. C., Schilizzi, R. T., Icke, V., Fejes, I., \& Spencer, R. E. 1987, Nature, 328, 309

Vermeulen, R. C., Schilizzi, R. T., Spencer, R. E., Romney, J. D., \& Fejes, I. 1993, A\&A, 270, 177 